\title{
GENE VS. LIFE STYLE: A 10-YEAR LONGITUDINAL STUDY DETERMINING MECHANISMS OF INDIVIDUAL DIFFERENCE IN AGE-RELATED ARTERIAL STIFFENING
}

\author{
Sugawara, J. ${ }^{1}$, Tomoto, T. ${ }^{1}$, Noda, N. $^{1}$, Matsukura, S. ${ }^{1}$, Tsukagoshi, K. ${ }^{1}$ and Maeda, S. ${ }^{2}$ \\ ${ }^{I}$ National Institute of Advance Industrial Science and Technology, Tsukuba, Japan \\ ${ }^{2}$ University of Tsukuba, Tsukuba, Japan
}

\begin{abstract}
Introduction
Increased arterial stiffness is a strong cardiovascular disease (CVD) risk (Vlachopoulos et al. 2010). Previous cross-sectional and short-term intervention studies suggest large inter-individual differences in magnitude of age-related arterial stiffening as well as efficiency of regular physical activity on arterial destiffening (Iemitsu et al. 2006; Hayashi et al. 2008). To shed light on underlying mechanisms responsible for individual difference in age-related arterial stiffening, we examined contributions of polymorphisms in endothelin (ET) and estrogen-related genes and regular physical activity using a 10-year longitudinal study design.
\end{abstract}

\section{Methods}

We evaluated changes in blood pressure and brachial-ankle pulse wave velocity (baPWV), an index of arterial stiffness, in 90 volunteers ( 51 men, current age $=63 \pm 14 \mathrm{yrs}$ ) who had participated other experiments including baPWV measurement in our laboratory approximately 10 years ago. We determined the gene single-nucleotide polymorphisms of $958 \mathrm{~A} / \mathrm{G}$ in exon 6 of ET-A receptor (ET-A), 831A/G in exon 4 of ET-B receptor (ET-B), and at $-401 \mathrm{~T} / \mathrm{C}$ of intron 1 of the estrogen receptor (ER)- $\alpha$ gene. Average follow-up duration was $10 \pm 0.9$ years. Subjects were divided into three groups based on current physical activity level (quantified by a questionnaire): less than 5 METs/week (LO, n=49), 5-15 METs/week (MID, n=23), and more than 15 METs/week (HI, $n=18$ ) of aerobic exercise.

\section{Results}

In pooled subjects, baPWV increased by $+11.8 \%$ (from $13.4 \pm 2.6$ to $14.9 \pm 3.1 \mathrm{~m} / \mathrm{sec}$ ). HI group exhibited significantly smaller increase in baPWV $(+5.5 \pm 9.9 \%)$ compared with the other groups (LO: $+13.4 \pm 10.7 \%$; MID; $+13.4 \pm 11.0 \%$ ) after adjustment for confounding factors (e.g., current age, current blood pressure, and baseline baPWV level), irrespective of each genotype. Interestingly, physically inactive individuals (i.e., LO and MID groups) having G/G genotype of ET-B showed significantly and substantially greater increase in baPWV $(19.1 \pm 12.9 \%)$ than the others having AA or A/G genotypes $(12.2 \pm 9.0 \%$ and $11.4 \pm 9.9 \%$, respectively) after adjustment for confounding factors. ET-A and ER- $\alpha$ genotype did not affect age-related increase in baPWV.

\section{Discussion}

The 10-year longitudinal observation establishes the attenuating effect of regular aerobic exercise on age-related arterial stiffening. In addition, our results suggest that ET-B polymorphism may influence extent of arterial stiffening with aging. Increased arterial stiffness has emerged as a strong predictor of future CVD events and all-cause mortality (Vlachopoulos et al. 2010). Therefore, to shed light on mechanisms responsible for divergent arterial stiffening with age is important to devise strategy of CVD prevention. The present study provides us important caution that specific polymorphisms of ET-related gene possibly facilitate arterial stiffening.

\section{References}

Iemitsu M, Maeda S, Otsuki T, et al. (2006). Polymorphism in endothelin-related genes limits exercise-induced decreases in arterial stiffness in older subjects. Hypertension, 47, 928-936.

Hayashi K, Maeda S, Iemitsu M, et al. (2008). Estrogen receptor- $\alpha$ genotype affects exercise-related reduction of arterial stiffness. Med Sci Sports Exerc, 40, 252-257.

Vlachopoulos C, Aznaouridis K, Stefanadis C. (2010). Prediction of cardiovascular events and all-cause mortality with arterial stiffness: A systematic review and meta-analysis. J Am Coll Cardiol, 55:1318-1327. 